\title{
Envy-free and efficient minimal rights: recursive no-envy*
}

\author{
Diego Domínguez ${ }^{\dagger}$ \\ Instituto Tecnológico Autónomo de México
}

Antonio Nicolò

University of Padova

This version, July 14, 2008

\footnotetext{
${ }^{*}$ This paper was presented at the 2008 Society for Social Choice Meetings in Montreal we are grateful for all comments from seminar participants.

${ }^{\dagger}$ Corresponding author. Camino Santa Teresa 930, México D.F., 10700, México. e-mail: ddomingue@itam.mx

†Via del Santo 33, 35123, Padova, Italy. e-mail: antonio.nicolo@unipd.it
} 


\begin{abstract}
In economics the main efficiency criterion is that of Pareto-optimality. For problems of distributing a social endowment a central notion of fairness is no-envy (each agent should receive a bundle at least as good, according to her own preferences, as any of the other agent's bundle). For most economies there are multiple allocations satisfying these two properties. We provide a procedure, based on distributional implications of these two properties, which selects a single allocation which is Pareto-optimal and satisfies no-envy in two-agent exchange economies. There is no straightforward generalization of our procedure to more than two-agents.
\end{abstract}

Keywords: no-envy, fair allocation, recursive methods, exchange economies JEL Classification Number: D63, D74 


\section{Recursive no-envy}

In the economics literature on fair allocation one central equity concept is no-envy (Foley 1967), each agent should receive a bundle at least as good, according to her own preferences, as any of the other agent's bundle 1 Starting with Varian (1974) different allocation rules have been proposed which select envy-free and efficient allocations in exchange economies 2 One proposal which selects an envy-free allocation is by means of an iterative procedure based on partial assignment of most preferred allocations within the set of envy-free allocations (Baumol 1982). Unfortunately, this procedure may fail to select an efficient allocation (Philpotts 1983). We propose a modification to Baumol's (1982) proposal, based on partial assignment of most preferred allocations within the set of efficient and envy-free allocations, and show that under mild conditions it selects an efficient and envy-free allocation for two-agent exchange economies.

Our rule is based on strengthening the no-envy condition in a recursive manner. First, we define the "minimal right of each agent" as the minimal amounts of commodities that she receives at any envy-free and efficient allocation. Then, if all members of society agree that an envy-free and efficient allocation should be selected, they implicitly agree that each agent should receive at least her minimal right and we can assign these to each agent. Once minimal rights have been assigned we can focus on distributing the resources remaining after such assignment. Then, it is only natural to try to distribute these resources in an envy-free and efficient way; but then we can apply the same argument calculate minimal rights, assigned them and proceed recursively until minimal rights are zero. We show that, for two-agent economies, (i) assigning to each agent her minimal right guarantees that the final allocation will be envy-free, and (ii) iterated assignment of minimal rights process leads to an (envy-free and) efficient allocation.

This result is in contrast with some previous results in the fair allocation literature where a society may start from an inefficient envy-free allocation, engage in "envy-free trades",

\footnotetext{
${ }^{1}$ When an allocation satisfies no-envy we say that it is an envy-free allocation.

${ }^{2}$ For a survey of the fair allocation literature see Thomson (2007).
} 
and end up in an efficient allocation where there is envy (Feldman and Kirman 1974). This result shows that sometimes "adding" to an initially fair allocation a fair transition, where the same notion of fairness is applied to the initial state and the transition, leads to an unfair final result. In our procedure this is not the case, we can think of each agent receiving her minimal right as a transition principle and our results show that recursively applying this transition principle leads to a fair final outcome. Each agent starts receiving nothing, which is a fair envy-free initial state; then, at each step of the process each agent receives a her minimal right which defines an envy-free allocation (thus, no agent could object to such assignment on the basis of envy) and is a fair transition; but then, the cumulative amounts received up to that step define a new initial state which we show it is envy-free in the original economy and thus a fair initial state to which we can apply the transition principle, our results show that in the limit we obtain a fair and efficient allocation.

The idea of distributing each agent's minimal rights and iterating this procedure until the entire endowment is assigned to the agents resembles the idea of a gradual process which is already present in the bargaining literature. The idea of gradualism in bargaining firstly appear in the seminal paper of Admati and Perry (1991). More recently, Compte and Jehiel (2003) presents a model in which gradualism derives from reciprocal concessions that agents make under the threat of inefficient termination option. For cake division problems Nicolò and Yu (forthcoming) propose, in a fair division game, an iterated version of the divide and choose rule, previously analzyed by Crawford (1977), in which an envy-free and efficient allocation is reached after a step by step procedure.

Our procedure can also be interpreted as a non-manipulability of a distribution rule with respect to assignment of minimal rights. Since both agents agree that the other should receive her minimal rights, when faced with a specific distribution problem an arbitrator can decide to apply a distribution rule directly, or to first assign to each agent her minimal right (over which there is no conflict of interest) and then apply the rule to the remaining resources. In order to avoid manipulability of the procedure it is desirable to ask that 
the rule selects the same allocation no matter which of these two options the arbitrator chooses; it is easy to see that our rule is the only rule which satisfies this non-manipulability property. In bakruptcy problems this invariance property is widely accepted (Aumann and Maschler 1985) it says that a rule should recommend the same awards when applied directly to a problem, or first assigning minimal rights and then applied to a reduced problem $3 \mathrm{In}$ bankruptcy invariance under minimal rights does not select a single award vector since in the second step minimal rights are not positive. 4

The fact that our rule selects an efficient allocation is obtained by considering only efficient allocations when defining minimal rights, it can be generalized for more than two agents. The fact that it selects an envy-free allocation is obtained by noting that, for two-agent economies, no-envy can be simplified to comparing each agent's bundle with the remaining resources, 5 and then, assigning minimal rights in the first step guarantees no-envy of the final allocation. For economies with more than two-agents this reasoning fails, envy (or lack of envy) by one agent depends not only on what she receives but on the distribution of the remaining resources among the remaining agents; moreover, after assigning minimal rights in the first step, envy may arise after distributing the remaining resources in the economy.

The paper is organized as follows. The next section contains the model, Section 2 contains the results for two-agent economies, and in Section 4 we conclude and discuss some possible extensions for economies with more than two agents.

\footnotetext{
${ }^{3}$ In bankruptcy an agent's minimal right is given by the amount of the resource not claimed by the other agents,

${ }^{4}$ The invariance property has been studied applied to other notions of rights by Domínguez and Thomson (2006) and Domínguez (2007) and found that for some properties it does select a single award vector.

${ }^{5}$ Efficiency implies that all the resources are distributed among the agents.
} 


\section{The model}

There is a social endowment $\Omega \in \mathbb{R}_{++}^{m}$ of $M=\{1, \ldots, m\}$ commodities to be distributed among a set $N=\{A, B\}$ of agents 6 Each agent can consume non-negative amounts of each commodity, for each agent $i \in N$ her consumption set is $X_{i}=\mathbb{R}_{+}^{m}$. We refer to agent $i$ 's vector of consumption $x_{i} \in X_{i}$ as her bundle. Each agent $i \in N$ has a continuous, strictly monotonic, and strictly convex preference relation $R_{i}$ over her consumption set.7 We assume that preferences satisfy the following decreasing marginal rate of substitution condition: Let $x, x^{\prime} \in X_{i}$ such that $x \neq x^{\prime}$, and $x \nsupseteq x^{\prime}$ and $x \not x^{\prime}$, let $p$ and $p^{\prime}$ denote any supporting prices of the upper contour sets of $R$ at $x$ and $x^{\prime}$ respectively. Then, for each commodity $j, k \in M$, such that $x_{j} \geq x_{j}^{\prime}$ and $x_{k} \leq x_{k}^{\prime}$, the relative prices between commodity $j$ and $k$ satisfy, $\frac{p_{j}}{p_{k}} \leq \frac{p_{j}^{\prime}}{p_{k}^{\prime}} 8$ The set of all such preferences is denoted $\mathcal{R}$. A profile of preferences is $R=\left(R_{A}, R_{B}\right) \in \mathcal{R}^{2}$, an economy is a pair $(R, \Omega) \in \mathcal{E}=\mathcal{R}^{2} \times \mathbb{R}_{++}^{m}$.

An allocation $x=\left(x_{A}, x_{B}\right) \in \times_{i \in N} X_{i}$ assigns to each agent a bundle $x_{i} \in X_{i}$. The set of all possible allocations is denoted $X=\times_{i \in N} X_{i}$. An allocation $x \in X$ is feasible for the economy $(R, \Omega)$ if $\sum_{i \in N} x_{i} \leq \Omega$, that is, the aggregate endowment is enough to assign to each agent her bundle. For each economy $(R, \Omega) \in \mathcal{E}$ we denote its set of feasible allocations $Z(R, \Omega)$. A feasible allocation $x \in Z(R, \Omega)$ is efficient for the economy $(R, \Omega)$ if there is no feasible allocation $x^{\prime} \in Z(R, \Omega)$ such that, for each $i \in N, x_{i}^{\prime} R_{i} x_{i}$, and for some $i \in N$, $x_{i}^{\prime} P_{i} x_{i}$. For each economy $(R, \Omega) \in \mathcal{E}$ we denote its set of efficient allocations $P(R, \Omega)$. For each agent $i \in N$ the projection of the set $P(R, \Omega)$ onto her consumption space is denoted $P_{i}(R, \Omega) \cdot 9$ it consists of all bundles $x_{i} \in X_{i}$ such that $\left(x_{i}, \Omega-x_{i}\right) \in P(R, \Omega) 10$

\footnotetext{
${ }^{6}$ The set $\mathbb{R}_{+}$is the set of non-negative reals and the set $\mathbb{R}_{++}$is the set of positive reals. Vector inequalities: $x \geqq y \Leftrightarrow$ for each $i \in N, x_{i} \geq y_{i} . x \geq y \Leftrightarrow x \geqq y$ and $x \neq y . x>y \Leftrightarrow$ for each $i \in N, x_{i}>y_{i}$.

${ }^{7}$ Given a preference relation $R$, we denote strict preference by $P$ and indifference by $I$.

${ }^{8}$ Even though decreasing marginal rates of substitution states that the greater-than relation between relative prices of commodity $j$ in terms of commodity $k$ is independent of the consumption levels of other commodities, the level of the relative prices may depend on the consumption levels of other commodities. A sufficient condition for preferences to satisfy decreasing marginal rates of substitution is that preferences are both convex and homothetic.

${ }^{9}$ Throughout the paper we use the following notation: Given a set of allocations $S \subset X$, the set $S_{i} \subset X_{i}$ denotes the projection of $S$ onto agent $i$ 's consumption space.

${ }^{10}$ Note that by strict monotonicity of preferences if an allocation is efficient it distributes the social endow-
} 
An allocation $x=\left(x_{A}, x_{B}\right)$ satisfies no-envy (or is envy-free) for the economy $(R, \Omega)$ if each agent is at least as well off consuming her bundle than consuming the other agent's bundle, that is, for each $i, j \in N, x_{i} R_{i} x_{j}$. For each economy $(R, \Omega) \in \mathcal{E}$ we denote its set of envy-free allocations $F(R, \Omega)$. The set of envy-free and efficient allocations is denoted $P F(R, \Omega)$ and consists of the intersection of the sets $P(R, \Omega)$ and $F(R, \Omega)$.

We are interested in recommending to each economy an allocation. An allocation rule (or just a rule) $\varphi: \mathcal{E} \rightarrow Z(R, \omega), 11$ is a function from the set of economies into its set of feasible allocations. A rule is efficient if for each economy it recommends an efficient allocation, it satisfies no-envy if for each economy it recommends an envy-free allocation.

\subsection{Preliminaries}

We now provide some preliminary results on envy-free and efficient allocations. Our first result is well-known in the literature and was shown by Varian (1974) 12

Remark 1. For each $(R, \Omega) \in \mathcal{E}$ the set of envy-free and efficient allocations is a non-empty and closed set.

Given decreasing marginal rates of substitution, starting from an efficient allocation and moving to another feasible allocation in which each agent obtains a bundle which is no larger nor smaller than her original bundle each of the agent's supporting prices move opposite directions. Thus, the new allocation can not be efficient and each agent's set of efficient bundles defines an increasing curve in consumption space.

Remark 2. For each $(R, \Omega) \in \mathcal{E}$, the efficient set defines an increasing curve in the Edgeworth box, that is, if two distinct allocations $\left(x_{A}, x_{B}\right),\left(x_{A}^{\prime}, x_{B}^{\prime}\right) \in P(R, \Omega)$ then, either (i) $x_{A} \geq x_{A}^{\prime}$, or (ii) $x_{A} \leq x_{A}^{\prime}$.

\footnotetext{
ment fully.

${ }^{11} \mathrm{We}$ ask that a rule selects a single feasible allocation, one possibility for generalizing our results to more than two agents is to consider allocation correspondences.

${ }^{12}$ For economies without production the Walrasian rule operated from an equal distribution of the endowment leads to an envy-free allocation (since all agents face the same budget set), and by the first welfare theorem this allocation is also efficient.
} 
Proof. Let $(R, \Omega) \in \mathcal{E}$, and by contradiction assume that there exists $\left(x_{A}, x_{B}\right),\left(x_{A}^{\prime}, x_{B}^{\prime}\right) \in$ $P(R, \Omega)$ such that $x_{A} \nsucceq x_{A}^{\prime}$ and $x_{A} \not \leq x_{A}^{\prime}$. Let $p, p^{\prime} \in \mathbb{R}_{++}^{m}$ denote some supporting prices of $R_{A}$ at $x_{A}$ and $x_{A}^{\prime}$ respectively, and $q, q^{\prime} \in \mathbb{R}_{++}^{m}$ be supporting prices of $R_{B}$ at $x_{B}$ and $x_{B}^{\prime}$ respectively. Since $\left(x_{A}, x_{B}\right)$ and $\left(x_{A}^{\prime}, x_{B}^{\prime}\right)$ are efficient, we can find supporting prices such that $p=q$ and $p^{\prime}=q^{\prime}$.

First we show that the relative prices between any two commodities that move in opposite directions from $x_{A}$ to $x_{A}^{\prime}$ remain the same. Let $j, k \in M$ be such that $x_{A j} \geq x_{A j}^{\prime}$ and $x_{A k} \leq x_{A k}^{\prime}$. By efficiency the full endowment is consumed, hence we have $x_{B j} \leq x_{B j}^{\prime}$ and $x_{B k} \geq x_{B k}^{\prime}$. By decreasing marginal rates of substitution, $\frac{p_{j}}{p_{k}} \leq \frac{p_{j}^{\prime}}{p_{k}^{\prime}}$ and $\frac{q_{j}^{\prime}}{q_{k}^{\prime}} \leq \frac{q_{j}}{q_{k}}$; by efficiency we know that $\frac{p_{j}}{p_{k}}=\frac{q_{j}}{q_{k}}$ and $\frac{p_{j}^{\prime}}{p_{k}^{\prime}}=\frac{q_{j}^{\prime}}{q_{k}^{\prime}}$. Thus, it has to be that $\frac{p_{j}}{p_{k}}=\frac{p_{j}^{\prime}}{p_{k}^{\prime}}=\frac{q_{j}^{\prime}}{q_{k}^{\prime}}=\frac{q_{j}}{q_{k}}$.

Now we show that the relative prices between any two commodities that move in the same direction from $x_{A}$ to $x_{A}^{\prime}$ remain the same. Let $k, l \in M$ be such that $x_{A k} \geq x_{A k}^{\prime}$ and $x_{A l} \geq x_{A l}^{\prime}$. Let $j$ be such that $x_{A j} \leq x_{A j}^{\prime}$ (such $j$ exists since $x_{A} \nsucceq x_{A}^{\prime}$ and $x_{A}^{\prime} \nsucceq x_{A}$ ). By the previous step $\frac{p_{j}}{p_{k}}=\frac{p_{j}^{\prime}}{p_{k}^{\prime}}$ and $\frac{p_{l}}{p_{j}}=\frac{p_{l}^{\prime}}{p_{j}^{\prime}}$. Multiplying both equations we get $\frac{p_{l}}{p_{k}}=\frac{p_{l}^{\prime}}{p_{k}^{\prime}}$.

Hence, supporting prices of $R_{A}$ at $x_{A}$ and $x_{A}^{\prime}$ are the same, that is $p=p^{\prime}=q=q^{\prime}$.

Finally, let $x_{A}^{\prime \prime} \in \operatorname{box}\left(x_{A}, x_{A}^{\prime}\right)$ be such that $x_{A}^{\prime \prime} \neq x_{A}$ and $x_{A}^{\prime \prime} I_{A} x_{A} 13$ Such $x_{A}^{\prime \prime}$ exists by continuity of preferences. By decreasing marginal rates of substitution and using the same argument as above moving from $x_{A}^{\prime \prime}$ to $x_{A}$ and $x_{A}^{\prime}$ respectively lead to changes in supporting prices in weakly opposite directions; but given the equality of such prices remain constant. Thus $x_{A} I_{A} x_{A}^{\prime \prime}$ and supporting prices of $R_{A}$ at $x_{A}^{\prime \prime}$ and $x_{A}^{\prime \prime}$ are the same, which contradicts strict convexity of preferences.

\section{Envy-free and efficient minimal rights}

Given an economy $(R, \Omega)$ no-envy and efficiency restrict the bundles that each agent can receive. For each agent $i \in N$, the set of potential envy-free and efficient bundles,

\footnotetext{
${ }^{13}$ For each $x, x^{\prime} \in \mathbb{R}^{M} M$ the set $\operatorname{box}\left(x, x^{\prime}\right)=\left\{y \in \mathbb{R}^{M}\right.$ : for each $\left.j \in M, \min \left\{x_{j}, x_{j}^{\prime}\right\} \leq y_{j} \leq \max \left\{x_{j}, x_{j}^{\prime}\right\}\right\}$.
} 


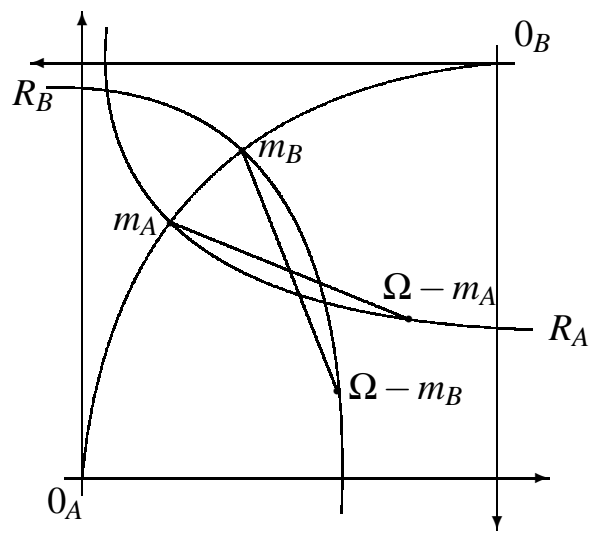

Figure 1: Minimal rights. The minimal rights of each agent $i$ are denoted $m_{i}$. Given decreasing marginal rates of substitution the Pareto set defines an increasing curve in the Edgeworth box. Each agent is indifferent between consuming her minimal right $m_{i}$ and the remaining resources $\left(\omega-m_{i}\right)$. Each agent's minimal right is part of an efficient allocation.

$P F_{i}(R, \Omega)$, consists on all bundles $x_{i} \in X_{i}$ such that $\left(x_{i}, \Omega-x_{i}\right) \in P F(R, \Omega)$. Each bundle $x_{i} \in P F_{i}(R, \Omega)$ requires different amounts of each commodity, the minimal rights of an agent are given by the minimum amounts of each commodity that she receives at any envy-free allocation (see figure 1).

Definition 1. For each $(R, \Omega) \in \mathcal{E}$, each $i \in N$ and each $k \in M$,

i. Agent i's minimal right of commodity $\mathbf{k}$ is $m_{i k}(R, \Omega)=\inf \left\{x_{k}\right.$ : there exists $x_{-k} \in$ $\left.\mathbb{R}_{+}^{M-1},\left(x_{k}, x_{-k}\right) \in P F_{i}(R, \Omega)\right\}$.

ii. Agent i's minimal rights are $m_{i}(R, \Omega)=\left(m_{i k}(R, \Omega)\right)_{k \in M}$.

iii. The economy's minimal rights are $m(R, \Omega)=\left(m_{i}(R, \Omega)\right)_{i \in N}$.

It is easy to see that for each economy its minimal rights define an envy-free and feasible allocation. As the next proposition shows, for each agent the allocation which assigns to that agent her minimal rights and the remainder to the other agent is an envy-free and efficient allocation (see Figure 1).

Proposition 1. For each $(R, \Omega) \in \mathcal{E}$ and each $i \in N$, the allocation $\left(m_{i}(R, \Omega), \Omega-m_{i}(R, \Omega)\right)$, is envy-free and efficient. 
Proof. Let $(R, \Omega) \in \mathcal{E}$ and $i \in N$. We need to show that $m_{i}(R, \Omega) \in P F_{i}(R, \Omega)$.

For each commodity $k \in M$ there exists a sequence of bundles $\left\{x_{i k}^{n}\right\}_{n \in \mathbb{N}} \in P F_{i}(R, \Omega)$ such that $x_{i k}^{n} \rightarrow m_{i k}(R, \Omega)$. Let $X(k)$ be the set of elements of such sequence and let $X=$ $\bigcup_{k \in M} X(k)$. By Proposition 2 the efficient set defines an increasing curve, therefore, we can order the elements of $X$ in a decreasing sequence, let $\left\{x^{n}\right\}_{n \in \mathbb{N}}$ be such decreasing sequence. Then, for each $n \in \mathbb{N}, x^{n} \in P F_{i}(R, \Omega)$ and $x^{n} \rightarrow m_{i}(R, \Omega)$. Since the set $P F_{i}(R, \Omega)$ is closed then $m_{i}(R, \Omega) \in P F_{i}(R, \Omega)$.

The intuition behind Proposition 1 is simple: since the efficient set defines an increasing curve in the Edgeworth box, agent $i$ 's minimal rights of each good are obtained from the same allocation, and therefore are part of an efficient and envy-free allocation.

The next corollary is one of the main results of the paper. If we assign to each agent her minimal rights then, no matter how we distribute the remaining resources, we obtain an envy-free allocation.

Corollary 1. For each $(R, \Omega) \in \mathcal{E}$ and each $x \in Z(R, \Omega)$, if $x \geq m(R, \Omega)$ then $x \in F(R, \Omega)$.

Proof. Let $(R, \Omega) \in \mathcal{E}, i \in N$, and $x \in Z(R, \Omega)$ such that $x \geq m(R, \Omega)$. Since $x \in Z(R, \Omega)$, by Proposition 1, $x_{j} \leq \Omega-x_{i}$. Moreover, since $x_{i} \geq m_{i}(R, \Omega)$ then $x_{j} \leq \Omega-m_{i}(R, \Omega)$. Hence,

$$
x_{i} R_{i} m_{i}(R, \Omega) R_{i}\left(\Omega-m_{i}(R, \Omega)\right) R_{i} x_{j}
$$

Thus, for each $i \in N$ we have $x_{i} R_{i} x_{j}$, and therefore $x \in F(R, \Omega)$.

The next corollary shows that, if in the economy there is a positive amount of resources, each agent's minimal rights are positive.

Corollary 2. For each $(R, \Omega) \in \mathcal{E}$ with $\Omega \geq 0,14$ and each $i \in N, m_{i}(R, \Omega) \geq 0$, moreover for each $i \in N$ there exists a commodity $k \in M$ such that $m_{i k}(R, \Omega) \geq \frac{\Omega_{k}}{2}>0$.

\footnotetext{
${ }^{14}$ The notation $\Omega \geq 0$ is used for $\Omega \geq(0, \ldots, 0)$.
} 
Proof. We show that $m_{i}(R, \Omega)=0 \Rightarrow \Omega=0$.

Let $(R, \Omega) \in \mathcal{E}$, and $i \in N$. By Proposition 1 we have $m_{i}(R, \Omega) R_{i}\left(\Omega-m_{i}(R, \Omega)\right)$. Suppose that $m_{i}(R, \Omega)=0$, then, $0 R_{i} \Omega$. By monotonicity of preferences $\Omega=0$.

To show that there exists a commodity $k \in M$ such that $m_{i k}(R, \Omega) \geq \frac{\Omega_{k}}{2}>0$, assume by contradiction that for each $k \in M$ with $\Omega_{k}>0, m_{i k}(R, \Omega)<\frac{\Omega_{k}}{2}$. Then $m_{i}(R, \Omega) \leq \frac{\Omega}{2}$ and by monotonicity of preferences the agent would prefer consuming the remaining resources $\left(\Omega-m_{i}(R, \Omega)\right) P_{i} m_{i}(R, \Omega)$ contradicting the conclusions of Proposition 1

If no-envy and efficiency are normative criteria shared by both agents then, in order to satisfy these two normative criteria, each agent must receive at least her minimal rights. Moreover, no matter how we distribute the remaining resources, assigning to each agent her minimal right guarantees an envy-free distribution. Hence, a natural way to select an allocation is to assign the minimal rights and then distribute the remaining resources. The remaining resources along with the agent's preferences over the remaining resources define a new economy, if the minimal rights of this economy are positive it is only natural to assign them and iterate the process until minimal rights are zero. In the next section we study such procedure.

\section{A selection form the no-envy and efficient set}

After assigning to each agent her minimal rights the remaining resources are given by $\left(\Omega-\sum_{i \in N} m_{i}(R, \Omega)\right) \in \mathbb{R}_{+}^{m}$, and the preferences of each agent over these remaining resources are the restriction of her preferences over bundles dominating her minimal rights. The remaining resources, and the iomplied preferences define a reduced economy (see Figure 2).

Definition 2. For each economy $(R, \Omega) \in \mathcal{E}$ the minimal rights reduced economy (or just reduced economy), $r^{m}(R, \Omega)$, is the economy $\left(R^{\prime}, \Omega^{\prime}\right) \in \mathcal{E}$, where:

(i) For each $i \in N$ and each $x_{i}, x_{i}^{\prime} \in X_{i}$ we have, $x_{i} R_{i}^{\prime} x_{i}^{\prime} \Leftrightarrow\left(x_{i}+y_{i}\right) R_{i}\left(x_{i}^{\prime}+m_{i}(R, \Omega)\right)$. 


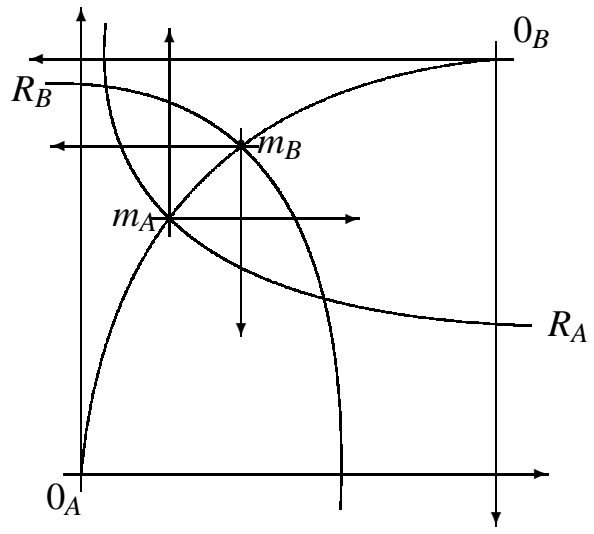

Figure 2: Minimal rights reduced economy. The economy's minimal rights are given by $\left(m_{A}, m_{B}\right)$. After assigning minimal rights we define the reduced economy given by the box $\left(m_{A}, m_{B}\right)$ and the implied preferences over the remaining resources.

(ii) $\Omega^{\prime}=\Omega-\sum_{i \in N} m_{i}(R, \Omega)$.

After assigning minimal rights, any efficient allocation in the reduced economy will also be an efficient allocation in the original economy. That is, if an allocation is efficient for the reduced economy then, after summing to each agent's bundle her minimal rights, we obtain an efficient allocation for the original economy.

Proposition 2. For each $(R, \Omega) \in \mathcal{E}$ an allocation $x \in P\left(r^{m}(R, \Omega)\right)$ if and only if the allocation $(m(R, \Omega)+x) \in P(R, \Omega)$.

Proof. The fact that if $(m(R, \Omega)+x) \in P(R, \Omega)$ then $x \in P\left(r^{m}(R, \Omega)\right)$ is straightforward. We show the converse holds.

Let $(R, \Omega) \in \mathcal{E},\left(R^{\prime}, \Omega^{\prime}\right)=r^{m}(R, \Omega)$, and $x \in P\left(R^{\prime}, \Omega^{\prime}\right)$. By contradiction assume that $(x+m(R, \Omega)) \notin P(R, \Omega)$. Then there exists an allocation $x^{\prime} \in P(R, \Omega)$ which Pareto-dominates $(x+m(R, \Omega))$ according to the preference profile $R$. By definition of $\Omega^{\prime}$ we have $\sum_{i \in N}\left(x_{i}^{\prime}-\right.$ $\left.m_{i}(R, \Omega)\right)=\Omega^{\prime}$, then, if for each $i \in N\left(x_{i}^{\prime}-m_{i}(R, \Omega)\right)>\geqq 0$ the allocation is $\left(x^{\prime}-m(R, \Omega)\right)$ is feasible in the reduced economy, and since by assumption $x^{\prime}$ Pareto-dominates $(x+$ $m(R, \Omega))$ according to the preference profile $R,\left(x^{\prime}-m(R, \Omega)\right)$ Pareto/dominates $x$ according to the preference profile $R^{\prime}$, but this contradicts the fact that $x \in P\left(R^{\prime}, \Omega^{\prime}\right)$, hence there 
exists an agent $i^{*} \in N$ and a commodity $k \in M$ such that $x_{i^{*} k}^{\prime}<m_{i^{*} k}(R, \Omega)$. By Proposition 1 $m_{i^{*}}(R, \Omega) \in P_{i^{*}}(R, \Omega)$ and by assumption $x_{i^{*}}^{\prime} \in P_{i^{*}}(R, \Omega)$; then, by Remark 2 , we have that $m_{*}(R, \Omega)>x_{*}^{\prime}$, and by monotonicity of preferences $\left(x_{i}+m_{*}(R, \Omega)\right) P_{i^{*}} x_{* i}^{\prime}$ contradicting the assumption that the allocation $x^{\prime}$ Pareto-dominates the allocation $(x+m(R, \Omega))$. Hence, $(x+m(R, \Omega)) \in P(R, \Omega)$

The intuition behind the proof is the following: if an allocation $x_{0}$ is efficient in the reduced economy and the sum of the allocation with the minimal rights $\left(x_{0}+m\right)$ is not efficient in the original economy, it must be Pareto-dominated by an allocation $x_{1}$ outside the Edgeworth box of the reduced economy. Since the efficient set defines an increasing curve in the Edgeworth box, the allocation $x_{1}$ gives to at least one agent a bundle smaller than her minimal rights, but this contradicts that $x_{1}$ Pareto-dominates $\left(x_{0}+m\right)$.

Once we assign to each agent her minimal rights, how should we assign the remaining resources? If there is a positive amount of resources remaining, minimal rights of the reduced economy are positive hence, a natural way of proceeding is to assign to each agent these new minimal rights and further reduce the economy, and iterate this procedure.

Definition 3. For each $(R, \Omega) \in \mathcal{E}$ and each $k \in \mathbb{N}$ we define the the k-envy-free and efficient minimal rights (or k-minimal rights) are defined recursively by:

$$
\begin{array}{r}
m^{1}(R, \Omega)=m(R, \Omega)+m\left(r^{m}(R, \Omega)\right) . \quad \text { fork }=1 . \\
m^{k}(R, \Omega)=m^{k-1}(R, \Omega)+m\left(r^{m^{k-1}}(R, \Omega)\right) 15 \text { fork } \geq 1 .
\end{array}
$$

Since minimal rights are positve, from Corollary 1 we know that for each agent the allocations assigning to the agent her $k$-minimal rights and the remainder to the other agent are envy-free, now we show that these allocations are also efficient.

Proposition 3. For each $(R, \Omega) \in \mathcal{E}$, each $i \in N$, and each $k \in \mathbb{N}$, the allocation $\left(m_{i}^{k}(R, \Omega), \Omega-\right.$ $\left.m_{i}^{k}(R, \Omega)\right)$ is envy-free and efficient. 
Proof. For each $j \in \mathbb{N}$ let $\left(R^{j}, \Omega^{j}\right)=r^{m^{j}}(R, \Omega)$. Let $K \in \mathbb{N}$, by Proposition 1 applied to the economy $\left(R^{K}, \Omega^{K}\right)$ we know that $\left(m_{i}\left(R^{K}, \Omega^{K}\right),\left(\Omega^{K}-m_{i}\left(R^{K}, \Omega^{K}\right)\right)\right)$ is efficient for the economy $\left(R^{K}, \Omega^{K}\right)$.

By Proposition 2 we have that $\left(\left(m_{i}\left(R^{K-1}, \Omega^{K-1}\right)+m_{i}\left(R^{K}, \Omega^{K}\right)\right),\left(m_{j}\left(R^{K-1}, \Omega^{K-1}\right)+\right.\right.$ $\left.\left.\Omega^{K}-m_{i}\left(R^{K}, \Omega^{K}\right)\right)\right)$ is efficient for the economy $\left(R^{k-1}, \Omega^{k-1}\right)$. Repeated application of Proposition 2] we have that $\left(\sum_{j=1}^{K} m_{i}\left(R^{j}, \Omega^{j}\right), \Omega-\sum_{j=1}^{K} m_{i}\left(R^{j}, \Omega^{j}\right)\right)$ is efficient for the economy $(R, \Omega)$, but by definition of the $k$-minimal rights we have that $\left(m_{i}^{K}(R, \Omega), \Omega-m_{i}^{K}(R, \Omega)\right)$.

Since minimal rights are positive the allocation $\left(m_{i}^{K}(R, \Omega), \Omega-m_{i}^{K}(R, \Omega)\right) \geq\left(m_{i}(R, \Omega), m_{j}(R, \Omega)\right)$, by Corollary 1 the allocation $\left(m_{i}^{K}(R, \Omega), \Omega-m_{i}^{K}(R, \Omega)\right)$ is envy-free.

Letting the process of assigning minimal rights continue we obtain an increasing sequence of feasible allocations, thus it has a limit. This limit defines the recursive minimal rights:

Definition 4. For each $(R, \Omega) \in \mathcal{E}$, the recursive envy-free and efficient minimal rights (or just recursive minimal rights) are given by:

$$
M(R, \Omega)=\lim _{k \rightarrow \infty} m^{k}(R, \Omega)
$$

Our main theorem shows that, not only does the results of Proposition 3 hold in the limit, but the recursive minimal rights define an efficient allocation.

Theorem 1. Recursive assignment of minimal rights selects an efficient and envy-free allocation.

Proof. Let $(R, \Omega) \in \mathcal{E}$.

First, we show that at the allocation $M(R, \Omega)$ all the resources are distributed, that is, $\sum_{i \in N} M_{i}(R, \Omega)=\Omega$. For each $k \in \mathbb{N}$, let $\left.\left(R^{k}, \Omega^{k}\right)=r^{m^{k}}(R, \Omega)\right)$, we need to show that $\Omega^{k} \rightarrow 0$. Since $\Omega^{k}=\Omega^{k-1}-\sum_{j \in N} m_{j}\left(r^{m^{k-1}}(R, \Omega)\right)$ it defines a decreasing sequence of non-negative vectors and it has a limit. Moreover, by Corollary 2 minimal rights are pos- 
itive and for each $i \in N$ we must have $m_{i}\left(r^{m^{k-1}}(R, \Omega)\right) \rightarrow 0$. By Corollary 2 there exists a commodity $l \in M$ such that $m_{i l}\left(R^{k}, \Omega^{k}\right) \geq \frac{\Omega_{l}^{k}}{2}$, but these two conditions imply that $\Omega_{k} \rightarrow 0$.

Now we show that $M(R, \Omega)$ is envy-free and efficient. Let $i \in N$, by the previous step $M(R, \Omega)=\lim _{k \rightarrow \infty}\left(m_{i}^{k}(R, \Omega), \Omega-m_{i}^{k}(R, \Omega)\right)$. By Proposition 3 for each $k \in \mathbb{N},\left(m_{i}^{k}(R, \Omega), \Omega-\right.$ $\left.m_{i}^{k}(R, \Omega)\right) \in P F(R, \Omega)$, and since the set of envy-free and efficient allocation is closed, $M(R, \Omega) \in P F(R, \Omega)$.

The intuition behind the proof of the main theorem is straightforward: Since the $k$ minimal rights are converging to the recursive minimal rights, then the minimal rights of the $k$-reduced economy must be converging to zero. Then, by Corollary 2 the endowment of the reduced economy must be going to zero, thus the recursive minimal rights distribute the entire resources. Now, since for each $k \in \mathbb{N}$ assigning to one agent her $k$-minimal rights and the remaining resources to the other agent is envy-free and efficient, then so is the limit.

\section{Conclusions}

For most economies there are many envy-free and efficient allocations. We provided a procedure which selects a single such allocation by strenghtening the concept of envy-free in a recursive manner. In this paper our focus is not strategic and we do not deal with the implementation problem; we offer a solution concept in which the step by step procedure is justified on a normative ground. For a given economy efficiency and envy-free properties identify a set of allocations. Hence, even if agents agree on the need for the final allocation to be efficient and envy-free, still they have the problem of selecting one allocation in this set. Our solution is parsimonius in that it does not rely on the agent's name or reference situations and it is only based on a logical consequence of agents' fairness concern: if agents agree on evaluating as socially desirable the properties of efficiency and envy-free, they cannot dispute over the amount of resources which is consumed by each agent in all efficient and envy-free allocations. Therefore, they should also agree on distributing this 
minimum amount, since it is a necessary condition for these two properties being satisfied. If agents do only agree on the normative relevance of the above mentioned properties, they can uprightly dispute on how to distribute any additional amount of resources. However, once these minimal rights are distributed, the economy has changed and now agents may agree on solving this new problem as they did before.

Generalizing our solution to economies with more than two agents has two main problems. First, envy-free is no longer defined by comparing what an agent consumes to the remaining resources, but depends on the distribution of the remaining resources among the rest of the agents. This problem can be solved by asking a weaker no-envy requirement based on an agent not envying the average amount of resources that the others receive. Second, is that for more than two agents the Pareto set no longer describes an increasing curve, and when defining minimal rights we can proceed in two ways: first, by selecting allocation which are minimal in terms of welfare (but then there may be multiplicity problems or we run into non-anonymous selections); second, by using the same definition of minimal rights in terms of commodities, but then we cannot guarantee the efficiency or envy-free of the selcted allocation. In many economic applications, such as economies with quasi-linear preferences, division of indivisible goods with monetary transfers (i.e. auction settings), or cake division problems, the Pareto set is well structured for any number of agents, in these settings our procedure can be generalized to acomodate more agents.

\section{References}

Admati, A., And M. Perry (1991): "Joint projects without commitment," Review of Economic Studies, 58, 259-276.

Aumann, R., AND M. MASChleR (1985): "Game theoretic analysis of a bankruptcy problem from the Talmud," Journal of Economic Theory, 36, 195-213. 
BAUMOL, W. (1982): “Applied fairness theory and rationing policy,” American Economic Review, 72, 639-651.

COMPTE, O., AND P. JEHIEL (2003): "Voluntary contributions to a joint project with asymmetric agents," Journal of Economic Theory, 112, 334-342.

Crawford, V. P. (1977): “A Game of Fair Division,” Review of Economic Studies, 44, 235-47.

Domínguez, D. (2007): "Lower bounds and recursive methods for the problem of adjudicating conflicting claims," mimeo.

Domínguez, D., And W. Thomson (2006): “A new solution to the problem of adjudicating conflicting claims," Economic Theory, 28, 283-307.

Feldman, A. M., And A. Kirman (1974): "Fairness and Envy," American Economic Review, 64(6), 995-1005.

FOLEY, D. (1967): "Resource allocation and the public sector," Yale Economic Essays, 7, 45-98.

Kolm, S.-C. (1972): “Justice et equité," Paris: Editions du Centre National de la Recherche Scientifique.

NiCOLÒ, A., AND Y. YU (forthcoming): "Strategic divide and choose," Games and Economic Behavior.

Philpotts, G. (1983): “Applied fairness theory: comment,” American Economic Review, $73,1157-1160$.

Thomson, W. (2007): "Fair allocation rules," Rochester center for economic research, working paper 539.

VARIAN, H. (1974): “Equity, envy and efficiency,” Journal of Economic Theory, 9, 63-91. 